\title{
Gravitational wave sensors based on superconducting transducers
}

\author{
Armen Gulian $\odot,{ }^{1,2,{ }^{*}}$ Joe Foreman, ${ }^{3}$ Vahan Nikoghosyan,,${ }^{1,4}$ Louis Sica, ${ }^{1}$ Pablo Abramian-Barco,${ }^{5}$ Jeff Tollaksen,, 2 \\ Gurgen Melkonyan, ${ }^{1}$ Iris Mowgood, ${ }^{1}$ Chris Burdette, ${ }^{1}$ Rajendra Dulal $\odot,{ }^{1,2}$ Serafim Teknowijoyo, ${ }^{1,2}$ Sara Chahid $\odot,{ }^{1,2}$ \\ and Shmuel Nussinov ${ }^{1,6}$ \\ ${ }^{1}$ Advanced Physics Laboratory, Institute for Quantum Studies, Chapman University, Burtonsville, Maryland 20866, USA \\ ${ }^{2}$ Schmid College of Science and Technology, Chapman University, Orange, California 92866, USA \\ ${ }^{3}$ Independent Researcher, Alexandria, Virginia 22310, USA \\ ${ }^{4}$ Institute for Physics Research, National Academy of Sciences, Ashtarak, 0203, Armenia \\ ${ }^{5}$ CIEMAT, Madrid 28040, Spain \\ ${ }^{6}$ School of Physics and Astronomy, Tel Aviv University, Ramat Aviv 69978, Tel Aviv, Israel
}

(Received 26 March 2021; accepted 15 October 2021; published 8 November 2021)

\begin{abstract}
Following the initial success of LIGO, new advances in gravitational wave (GW) detector systems are planned to reach fruition during the next decades. These systems are interferometric and large. Here we suggest different, more compact detectors of GW radiation with competitive sensitivity. These nonresonant detectors are not interferometric. They use superconducting Cooper pairs in a magnetic field to transform mechanical motion induced by GW into detectable magnetic flux. The detectors can be oriented relative to the source of GW, so as to maximize the signal output and help determine the direction of nontransient sources. In this design an incident GW rotates infinitesimally a system of massive barbells and superconducting frames attached to them. This last rotation relative to a strong magnetic field generates a signal of superconducting currents. The suggested arrangement of superconducting signal sources facilitates rejection of noise due to stray electromagnetic fields. In addition to signal analysis, we provide estimates of mechanical noise of the detector, taking into account temperature and elastic properties of the loops and barbells. We analyze at which parameters of the system a competitive strain sensitivity could be achieved. We have tested the basic idea of the detector in the laboratory and reached the theoretical Johnson-Nyquist noise limit with multiturn coils of normal metal. Realization of full-blown superconducting detectors can serve as viable alternatives to interferometric devices.
\end{abstract}

DOI: 10.1103/PhysRevResearch.3.043098

\section{INTRODUCTION}

In 1962, Gertsenshtein and Pustovoit suggested using a photonic interferometer for gravitational wave (GW) detection [1]. It took more than half-century to successfully realize this idea in the LIGO instruments by a large international team of researchers $[2,3]$ opening thus a new era in astrophysics. To further facilitate progress in this direction, higher sensitivity instruments are required. Currently, various systems more advanced than LIGO are projected (see Table I).

They are interferometric and require long optical arm lengths and/or cryogenic cooling. We suggest here that more compact and still higher sensitivity instruments can be realized with the help of the unique features of superconductivity and superconducting electronics. Our instruments, unlike Weber's cylinders [12-14] or LIGO mirrors [15], utilize not the longitudinal motion caused by the GW, which at a distance $L$

\footnotetext{
*Corresponding author: gulian@chapman.edu

Published by the American Physical Society under the terms of the Creative Commons Attribution 4.0 International license. Further distribution of this work must maintain attribution to the author(s) and the published article's title, journal citation, and DOI.
}

between the mechanical parts move by $\delta L \propto L h_{\mathrm{GW}}$, but rather the shift of rotational angle: $\delta \Theta \propto h_{\mathrm{GW}}$. Rotational motion allows us to transfer GW energy into mechanical energy, and then convert it into an electric signal using principles different from the conventionally used optical readout.

\section{A. Ultimate energy resolution limit}

Transferring certain GW energy into mechanical energy is an important first step in the operation of any detector. Giffard derived already in 1976 [16] a criterion for maximum sensitivity of linear motion [17] GW detectors for the action of GW pulses on these detectors. If the detector is equipped with a sensor having an ultimate energy resolution limit (ERL), which is $E_{R} \approx \hbar$ [18], then, for maximum sensitivity, a pulse with a characteristic Fourier component $\omega_{\mathrm{GW}}$ should be capable of driving the antenna from rest to a mechanical energy level exceeding

$$
E_{m} \geqslant 2 \hbar \omega_{\mathrm{GW}} \text {. }
$$

Giffard's criterion is derived for resonant detectors. Equation (1) can be considered approximately valid also for a wideband detector, with $\omega_{\mathrm{GW}}$ being a proper average value of the sensitive frequency band [19]. The LIGO detector, for example, satisfies this criterion (1). Indeed, substituting 
TABLE I. Some of the GW detectors targeting sensitivity beyond LIGO.

\begin{tabular}{|c|c|c|c|}
\hline Name [Ref.] & Design & Sensitivity (bandwidth) & Location, year of completion \\
\hline KAGRA [4] & $\begin{array}{l}\text { Interferometer, } \\
\text { cryogenic, } 3 \mathrm{~km}\end{array}$ & $\begin{array}{c}2 \times 10^{-24} / \mathrm{Hz}^{1 / 2} \\
(@ 100 \mathrm{~Hz})\end{array}$ & Japan, 2019 \\
\hline Einstein & Interferometer, & $5 \times 10^{-25} / \mathrm{Hz}^{1 / 2}$ & Europe \\
\hline Telescope [5] & $10 \mathrm{~km}$ & (@100 Hz) & \\
\hline Cosmic & Interferometer, & $2 \times 10^{-25} / \mathrm{Hz}^{1 / 2}$ & USA, $\sim 2030$ \\
\hline Explorer [6] & $40 \mathrm{~km}$ & (@100 Hz) & \\
\hline $\begin{array}{l}\text { Advanced+ } \\
\text { LIGO [7] }\end{array}$ & $\begin{array}{l}\text { Interferometer, } \\
\quad 4 \mathrm{~km}\end{array}$ & $\begin{array}{c}5 \times 10^{-25} / \mathrm{Hz}^{1 / 2} \\
(@ 200 \mathrm{~Hz})\end{array}$ & USA, 2023 \\
\hline $\begin{array}{l}\text { Advanced+ } \\
\text { VIRGO [8] }\end{array}$ & $\begin{array}{l}\text { Interferometer, } \\
\quad 3 \mathrm{~km}\end{array}$ & $\begin{array}{c}5 \times 10^{-25} / \mathrm{Hz}^{1 / 2} \\
(@ 200 \mathrm{~Hz})\end{array}$ & Europe, 2023 \\
\hline DECIGO $[9,10]$ & $\begin{array}{l}\text { Interferometer, } \\
1000 \mathrm{~km}\end{array}$ & $\begin{array}{c}4 \times 10^{-21} / \mathrm{Hz}^{1 / 2} \\
(@ 0.1 \mathrm{~Hz})\end{array}$ & Japan, 2024 \\
\hline LISA [11] & $\begin{array}{l}\text { Interferometer, } \\
2.5 \mathrm{mln} \mathrm{km}\end{array}$ & $\begin{array}{c}6 \times 10^{-22} / \mathrm{Hz}^{1 / 2} \\
(@ 0.005 \mathrm{~Hz})\end{array}$ & Europe, 2034 \\
\hline
\end{tabular}

into $E_{m} \sim M\left(\omega_{\mathrm{GW}} L h_{\mathrm{GW}}\right)^{2}$ the mirrors mass $M_{\mathrm{LIGO}} \sim 100 \mathrm{~kg}$, the lateral size $L_{\mathrm{LIGO}} \sim 2 \mathrm{~km}$, the characteristic frequency $\omega_{\mathrm{GW}} \sim 2 \pi \times 10^{2} \mathrm{~s}^{-1}$, and the detected GW amplitude $h_{\mathrm{GW}} \sim$ $10^{-21}$, we arrive at the estimate $E_{m} \sim 10^{-28} \mathrm{~J}$, which exceeds the value $2 \hbar \omega_{\mathrm{GW}} \sim 10^{-31} \mathrm{~J}$. This comparison tells us that LIGO-type detectors in principle can be advanced to higher sensitivity since they are still far from the limit outlined by (1). With the condition (1) one can calculate the ultimate performance of any linear motion GW detector, including our detector.

\section{B. The underlying concept of our detector}

The detector consists of two, mechanically separate, barbells. Each barbell is connected to a superconducting loop, so that under the action of periodic GW these loops rotationally oscillate towards or away from each other. Due to this rotational motion, magnetic fluxes of opposite signs are intercepted by the loops and transferred to SQUIDs for detection (Fig. 1). Following the logical scheme of the detector operation: transformation of gravitational wave energy (GE) into magnetic-flux energy (FE) via a superconducting transducer (ST), we call it the "GEFEST" detector.

\section{The detector resolution limits}

Let us explore the mechanical parameters of the detector that achieve the ultimate energy resolution. For a purepolarized GW [23] incident along the $\mathrm{z}$ axis, the system of coordinates can always be oriented as shown in Fig. 2 .

In a locally nearly flat space-time with the origin of the $(x, y, z)$ coordinate system coinciding with the center of mass of the barbells, the GW force acting on a particle with mass $m$ at location $r=\left(x_{0}, y_{0}\right)$ induces a tidal acceleration $a_{\mathrm{GW}}^{i}$. This can be found from the geodesic deviation [22]

$$
F_{\mathrm{GW}}^{i}(\mathbf{r} ; t) / m \equiv a_{\mathrm{GW}}^{i}(\mathbf{r} ; t)=-R_{0 j 0}^{i} r^{j},
$$

where $i, j=(x, y)$, and the relevant components of the Riemann tensor are given in terms of the transverse traceless perturbation of the metric

$$
\begin{gathered}
R_{0 x 0}^{x}=-h_{x x ; 00}^{T T} / 2=-R_{0 y 0}^{y}=\omega^{2} h_{x x}^{T T} / 2, \\
R_{0 x 0}^{y}=R_{0 y 0}^{x}=0 .
\end{gathered}
$$

Equations (3) and (4) are written assuming a harmonic GW with frequency $\omega: h_{x x}^{T T}=h_{\mathrm{GW}} \cos (\omega t)$, so that $a_{\mathrm{GW}}^{x}(\mathbf{r} ; t)=-\left(\omega^{2} x_{0} h_{\mathrm{GW}} / 2\right) \cos (\omega t)$, and $a_{\mathrm{GW}}^{y}(\mathbf{r} ; t)=$ $-\left(\omega^{2} y_{0} h_{\mathrm{GW}} / 2\right) \cos (\omega t)$. Because of this acceleration, the GW exerts a torque on the barbells. In the optimal barbell arrangement with the angle $\theta=\pi / 4$ shown in Fig. 2, the torque is $\tau_{z}^{\mathrm{GW}}=M \omega^{2} h_{\mathrm{GW}} \cos (\omega t) r^{2}$, where $M$ is the mass of each barbell sphere (half of the total barbell mass with the mass of the

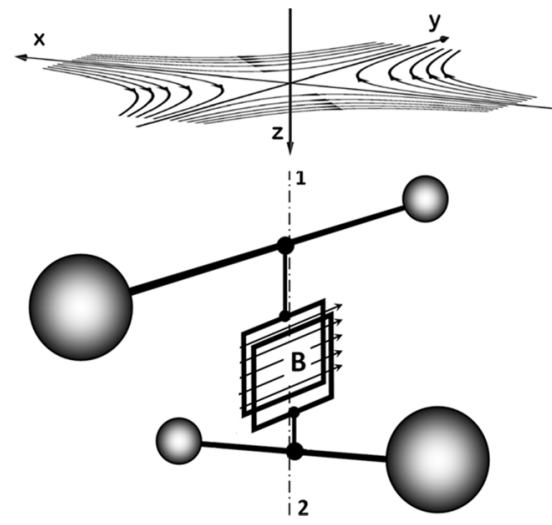

FIG. 1. Two orthogonally-oriented and mechanically separate barbells under the influence of an incident plane periodic GW oscillate around the axis (1-2). The direction of $\mathrm{GW}$ is assumed to coincide with this axis. The GW field-lines [20-22] (see also Fig. 2 below) are indicated for this case. Both barbells are rigidly connected with the dedicated superconducting loops (their rectangular shapes are adopted in the current layout). The magnetic field $\mathbf{B}$ is static in space and time. In absence of GW, the magnetic-field lines are in parallel to the loop planes, and no current is flowing through the loop. Upon the action of GW, the barbells' oscillatory rotation causes the superconducting loops to rotate in opposite directions, thus generating opposite polarity currents in the superconducting loops. 


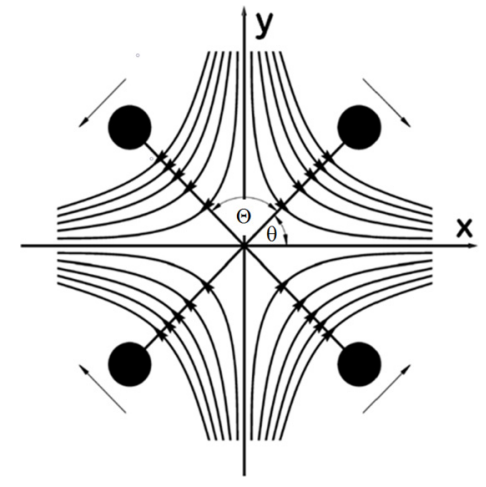

FIG. 2. The acceleration field lines of planar wavefront, purely polarized ("+" or " $x$ ") GW are indicated by the sets of hyperbolas. The direction of accelerations is shown by arrows for a given halfperiod of the wave; in the next half-period they will be reversed. A mechanical system of 2 point masses connected by a rigid line is a barbell with nonzero quadrupolar momentum. Two such barbells will oscillate with respect to each other. Angular amplitude of relative oscillation $\delta \Theta$ will constitute twice the value of the amplitude $\delta \theta$ of single barbell oscillation relative to a nonmoving observer associated with $(x, y)$ coordinate system and with the magnetic field $\mathbf{B}$ in Fig. 1.

connecting rod neglected). Since the moment of inertia of the barbell is $I=2 M r^{2}$ (where $r$ is the half-length of the barbell rod, i.e., the distance from the axis of rotation $z$ to the center of the barbel sphere), the angular velocity $\Omega=d(\delta \theta) / d t$ can be found from the equation of motion

$$
\frac{d \Omega}{d t}=\frac{\tau_{z}^{\mathrm{GW}}}{I}, \text { i.e., } \frac{d^{2}(\delta \theta)}{d t^{2}}=\frac{1}{2} \omega^{2} h_{\mathrm{GW}} \cos (\omega t),
$$

or, after integration,

$$
\delta \theta(t)=-\frac{1}{2} h_{\mathrm{GW}} \cos (\omega t) .
$$

Hitherto it was tacitly assumed that the presence of the finite distributed masses of the detector does not affect the GW field appearing in Eqs. (3) and (4). This is expected from General Relativity, since the GW-field can always be expressed via the Weyl tensor, thus constituting that part of Riemann's tensor, which does not depend locally on the mass distribution [24,25]. In view of relation (5) and (6), the total kinetic energy of two barbells is

$$
E_{\mathrm{kin}}=2 \times I \frac{\Omega^{2}}{2}=M \frac{\omega^{2}}{2} r^{2} h_{\mathrm{GW}}^{2} \sin ^{2}(\omega t) .
$$

To transfer the maximum amount of energy, the barbell arms should be as large as possible, with maximal individual masses.

The torque on the circuit loop from the attached barbell leads to its rotation in the magnetic field and a resultant electrical energy. The barbell torque, however, is opposed by a counter torque consistent with Lenz's law and resulting from the direction of the loop-current flow. This counter torque produces a mechanical perturbation that should be able to travel at the speed of sound $u$ over the distance $r+R$ ( $R$ the radius of the barbell sphere) during the half-period $t=\pi / \omega$ of the GW. Therefore, the maximum size of the barbell is
TABLE II. Expected parameters of proposed detector with specified sizes in case of Giffard's ultimate energy resolution limit.

\begin{tabular}{lccc}
\hline \hline$f(\mathrm{~Hz})$ & ERL $(\mathrm{J})$ & $r(\mathrm{~km})$ & $h_{\mathrm{GW}}$ (dimensionless) \\
\hline 100 & $1.26 \times 10^{-31}$ & 0.03 & $8.4 \times 10^{-23}$ \\
10 & $1.26 \times 10^{-32}$ & 0.3 & $2.6 \times 10^{-23}$ \\
1 & $1.26 \times 10^{-33}$ & 3 & $8.4 \times 10^{-24}$ \\
0.1 & $1.26 \times 10^{-34}$ & 30 & $2.6 \times 10^{-24}$ \\
0.01 & $1.26 \times 10^{-35}$ & 300 & $8.4 \times 10^{-25}$ \\
0.001 & $1.26 \times 10^{-36}$ & 3000 & $2.6 \times 10^{-25}$ \\
\hline \hline
\end{tabular}

determined by

$$
(r+R) \leqslant \frac{\pi u}{\omega} .
$$

Choosing barbell spheres made of $P b$ with the density $11343 \mathrm{~kg} / \mathrm{m}^{3}$, so that a sphere of radius $R=1.28 \mathrm{~m}$ has a mass of 100 ton (which is not a principal limitation!) we arrive at the estimates shown in Table II.

One should make a very important remark here. In the estimates above, the longitudinal speed of sound $u$ was used. However, for the simple geometry shown in Fig. 1, one should expect a flexural resonant frequency, which is much lower than the longitudinal one. Roughly, the relevant speed of sound for flexural deformations would be reduced by a factor $r_{a} / r$ with respect to the longitudinal velocity, where $r_{a}$ is the radius of the arm's cross section, and $r$ the arm length. Obviously, $r_{a} \ll r$, which will significantly reduce the speed of propagation of mechanical action from the barbell spheres, correspondingly reducing the resonant frequency of the system, and spoil the sensitivity of the proposed detector. To avoid this serious limitation, one should consider a more sophisticated design shown in Fig. 3.

As shown in Fig. 3(b), this design utilizes the rigidity of a triangular shape, which yields longitudinal deformation along the barbell arms. The flexural deformation will occur only in
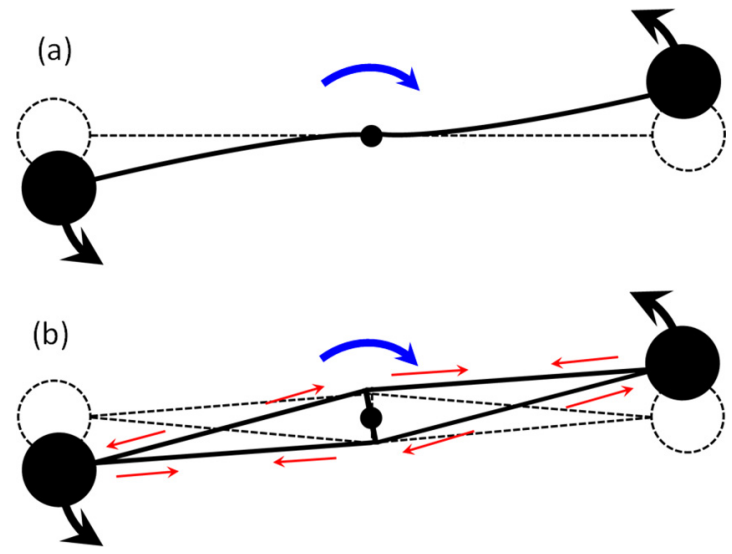

FIG. 3. Converting flexural deformations into longitudinal. (a) The counter action of GW-induced torques (corresponding forces shown by black arrows) and magnetic feedback-caused torque (blue arrow) leads to flexural deformations. (b) The triangular geometry converts the flexural deformation into the longitudinal deformation in the long arms. Red arrows indicate stretching and expanding deformations of the long arms of the barbells. 
the base of the triangle. However, this base can be very short, with the length $r_{b} \simeq r_{a}$, i.e., without compromising the disturbance propagation speed. The shape of triangle with very large aspect ratio $r / r_{b}$ is only possible because of the extremely weak forces induced by GWs (otherwise, the rigidity of the triangle will require larger values of $r_{b}$ ). This design eliminates flexural deformation in the plane of the barbell rotation. Its 3D generalization (pyramid) will eliminate also possible spontaneous flexural deformation along the orthogonal axis. This kind of "architectural" design should be used in practical implementation of the detector. However, for estimating its basic parameters the idealized model of a single-rod barbell will be used throughout this article. In constructing Table II, the value of $r$ was obtained from (8) where $R \ll r$ is neglected and $u=6 \times 10^{5} \mathrm{~cm} / \mathrm{s}$ was adopted. The value of $h_{\mathrm{GW}}$ was obtained by equating $E_{\text {kin }}$ of Eq. (7) with $E_{m}$ of Eq. (1), i.e., assuming the energy sensor provides the ultimate ERL, and no other noise sources are involved. Then in all cases given in Table II, the strain sensitivity is $\hat{h}_{\mathrm{GW}}=8.4 \times 10^{-24} \mathrm{~Hz}^{-1 / 2}$. It can be further improved by increasing, the hitherto unconstrained, mass $M$ beyond the 100 -ton limit $\left(\hat{h}_{\mathrm{GW}} \propto 1 / M^{1 / 2}\right)$. While some of the parameters in Table II are unrealistic from the point of view of contemporary space technologies, the attractiveness of the outlined sensitivity $\hat{h}_{\mathrm{GW}}$ warrants further exploration of the design and performance of these detectors, which will be carried out below.

\section{ELECTRONIC MOTION GENERATED BY GRAVITATIONAL WAVES}

The rotational motion has been used in various gravity gradiometers [26-29] and suggested for GW detectors in the past by the Braginsky group [30,31], and by Sakharov [32]. Recently, the rotational design for GW detectors was revived by the TOBA group [33] and simultaneously and independently by our group [34]. While TOBA utilizes optical interferometry for detecting the rotational motion, in our design the mechanical motion is converted into the motion of Cooper condensate to be detected via superconducting electronics.

The idea to register the action of GW via the electronic motion directly seems appealing, especially if this motion is performed by very "quiet" Cooper pairs in superconductors, while the ionic system stays immobile. One possible conceived realization of this idea was to use the different effective masses of electrons in different metals [34]. However, because of exact momentum conservation in inertial experiments, the effective mass concept does not work for inertial excitation [35], including the action of gravitational forces [36,37]. Furthermore, although single-particle excitations such as electrons and holes may have different effective masses in various superconductors, it is recognized in the superconductivity community that Cooper pairs have a mass, which equals twice the bare electron mass with very high accuracy [38]. Other possibilities for the generation of electronic motion by $\mathrm{GW}$ action [39] use the Tolman-Stuart mechanism [40] or the change of chemical potentials of metals under compression [41,42]. However, in both cases the back-reaction effects related to the induced magnetic fields drastically reduce the conversion efficiency $[39,43,44]$ as happens also for the London moment $[45,46]$. We therefore used in the present design the magnetic field itself as a driving force in order to transfer the mechanical action of GW into the electronic motion in superconductors. This can be done using Faraday's induction via interception of magnetic flux by rotating a closed superconducting loop [47].

\section{A. Quantitative estimates of the generated flux}

As indicated in Fig. 2, two flat, rectangular, superconducting loops with area vectors $\mathbf{A}_{+}$and $\mathbf{A}_{-}$are placed in a constant homogeneous magnetic field $\mathbf{B}$. The flux through a loop is then

$$
\Phi_{ \pm}(t)=\mathbf{B} \cdot \mathbf{A}_{ \pm}=|B||A| \cos [\pi / 2 \mp \delta \theta(t)],
$$

where $[\pi / 2 \mp \delta \theta(t)]$ is the angle between the $\mathbf{A}_{ \pm}$and the $\mathbf{B}$ vectors [these vectors are orthogonal in the absence of GW, when $\delta \theta \equiv 0$, and the flux $\Phi(t)=0]$. When $\delta \theta \neq 0$, then the intercepted magnetic flux is

$$
\delta \Phi(t)=B A \delta \theta(t) .
$$

From hereon, we consider the response of the loop with the vector $\mathbf{A}_{+}=\mathbf{A}$ and the response of the loop corresponding to $\mathbf{A}_{-}=-\mathbf{A}$ is just its negative [48]. As can be derived using the Eqs. (2)-(6), the torque imposed on each of the barbells shown in Fig. 2 is

$$
\begin{aligned}
\tau_{z}(r, \theta) & =x_{0} F_{y}-y_{0} F_{x}=-r F \sin (2 \theta) \\
& =-I \omega^{2} h_{\mathrm{GW}} \sin (2 \theta) \cos (\omega t) / 2 .
\end{aligned}
$$

This torque depends on the angle $\theta$ and is maximal at $\theta=\pi / 4$ orientation, which is assumed in further calculations.

Once $\delta \theta \neq 0$, a magnetic flux is intercepted by the superconducting loop, which generates a current in the loop and induces a magnetic moment $\mathbf{M}=J \mathbf{A}$. This magnetic moment interacts with the $\mathbf{B}$ field and in turn creates a counterrotational feedback torque $\tau^{f . b .}=\mathbf{M} \times \mathbf{B}$. In the laboratory frame of reference where the magnetic field is at rest, the equation of motion of the barbell and connected current loop is [49]

$$
\begin{aligned}
\frac{d^{2}(\delta \theta)}{d t^{2}} & =\frac{\tau_{z}^{\mathrm{GW}}+\tau^{f . b .}}{I} \\
& =-\frac{1}{2} \omega^{2} h_{\mathrm{GW}} \cos (\omega t)+\frac{J A B \sin (2 \theta)}{I},
\end{aligned}
$$

where $J=J(\delta \theta)$ is the current through the superconducting loop. As above, we will assume that the detector, while executing harmonic oscillations with negligible phase delay, is oriented for receiving the maximum signal, i.e., $\langle\theta\rangle=\pi / 4$, where $\langle\ldots\rangle$ means the time average. Then, since $\delta \theta \equiv \theta-$ $\langle\theta\rangle \ll 1, \sin (2 \theta)=1$ with very high accuracy, and the angular dependence in the last term of (12) should be retained in $J(\delta \theta)$ only:

$$
\omega^{2} \delta \theta(t)=\frac{1}{2} \omega^{2} h_{\mathrm{GW}} \cos (\omega t)-\frac{a^{2} B}{I} J(\delta \theta),
$$

where $a=\sqrt{A}$ is the linear size of the loop. From this equation it follows that the magnetic feedback is negligible only if the moment of inertia is large enough:

$$
I \gg \frac{2 J a^{2} B}{\omega^{2} h_{\mathrm{GW}}} \text {. }
$$


Assuming this condition is fulfilled, the maximum attainable $\delta \theta$ from (13) is

$$
\delta \theta=h_{\mathrm{GW}} / 2
$$

and the amplitude $\delta \Phi$ has a value

$$
\delta \Phi=B a^{2} h_{\mathrm{GW}} / 2 .
$$

Then the maximal current in the superconducting loop is

$$
J=\frac{\delta \Phi}{L_{\text {circuit }}}=\frac{B a^{2} \delta \theta}{L_{\text {circuit }}}=\frac{B a^{2} h_{\mathrm{GW}}}{2 L_{\text {circuit }}},
$$

where $L_{\text {circuit }}$ is the total inductance of the loop including the circuit, which couples it to the flux detector. Substituting (17) into (14) we find that the detector response is maximal if

$$
I \gg \frac{a^{4} B^{2}}{\omega^{2} L_{\text {circuit }}} .
$$

We also notice that under these conditions, the resonant frequency of the system, which follows from (13) subject to (17) is given by

$$
\omega^{2}=\frac{B^{2} a^{4}}{2 r^{2} M L_{\text {circuit }}} .
$$

Below this frequency the sensitivity of the detector degrades, which is typical for oscillator-based GW detectors.

\section{B. Inductive energy}

In superconducting electronics, the coupling with the detector is accomplished by using a coupling coil. The impedance matching requires this coil to have the same inductance as the generating loop: $L_{\text {coil }}=L_{\text {loop }}$, so that $L_{\text {circuit }}=$ $2 L_{\text {loop. }}$. Thus the inductive energy being transferred to the detector (for example, to a SQUID) via the coil is

$$
E_{\text {ind }}=\frac{1}{2} L_{\text {coil }} J^{2} .
$$

Substituting (16) and (17), we find

$$
E_{\text {ind }}=\frac{1}{2} L_{\text {coil }} \frac{(\delta \Phi)^{2}}{\left(L_{\text {circuit }}\right)^{2}}=\frac{1}{8 L_{\text {loop }}} \frac{a^{4}\left(B h_{\mathrm{GW}}\right)^{2}}{4} .
$$

The inductance of a square loop is [50,51]:

$$
L_{\text {loop }}=\left(2 \mu_{0} / \pi\right) a[\ln (a / d)-0.774],
$$

where $\mu_{0}=4 \pi \times 10^{-7} \mathrm{H} / \mathrm{m}$ is vacuum permeability and $d$ is the wire diameter. Taking into account that currents are being generated in two loops, the total inductive energy is

$$
E_{\text {ind }}^{\text {tot }}=\frac{\pi a^{3}\left(B h_{\mathrm{GW}}\right)^{2}}{32 \mu_{0}[\ln (a / d)-0.774]} .
$$

The corresponding flux is twice the value provided by (16), so that the flux per square root of bandwidth is

$$
\delta \Phi / \sqrt{f}=B A \hat{h}_{\mathrm{GW}}, \quad \hat{h}_{\mathrm{GW}} \equiv h_{\mathrm{GW}} / \sqrt{f} .
$$

To be detectable, this signal should exceed the noise of the detector. This will be considered next.

\section{INTRINSIC NOISE OF THE DETECTOR}

The barbell, as well as the superconducting loop on its supporting frame, has finite elasticity and frequency-dependent dissipation. The fluctuation-dissipation theorem [52] relates dissipation to fluctuations at thermodynamic equilibrium. This mechanical noise of thermal origin is considered to be fundamental, because it directly competes with the GW signal and is independent of the readout method used. As such, this topic is well addressed in various GW detector proposals. The closest to our case is the treatment provided for the TOBA detector with rotating bars [33], certain aspects of which, after appropriate modifications, can be adopted to our case.

\section{A. Thermodynamically inevitable noise sources}

We will now consider certain noise channels in general, and then estimate their contributions for a specific implementable case.

\section{Thermomechanical fluctuations of the barbells}

Taking into account that in our case the mass of barbells is mainly localized in spheres rather than distributed evenly as in TOBA, we find that the spectral density of thermo-elastomechanical angular fluctuations is $[15,53]$

$$
\delta \theta_{\mathrm{mech}}^{b}(\omega)=\frac{\sqrt{8}}{r \omega_{b}} \sqrt{\frac{\phi_{\mathrm{mech}}^{b} k_{B} T^{b}}{M_{b} \omega}} .
$$

where $\phi_{\text {mech }}^{b}$ is the mechanical loss angle, $T^{b}$ the barbell temperature, $M_{b}$ the mass of the barbell, $\omega_{b}$ the lowest mechanical angular resonant frequency, which we will approximate as $\omega_{b}=\pi u_{b} / r$, with $u_{b}$ is the speed of sound in the material of the barbell's arm. In writing Eq. (25) we implicitly assumed that the frequency $\omega$ is below the lowest resonant frequency of the barbell, and the other resonant frequencies are much higher so as to avoid their contribution [15,53]. Equation (25) is quite similar to that in TOBA design [33] though it has a different numerical coefficient.

\section{Thermomechanical fluctuations of the frame}

Fluctuations in the superconducting loop frames are quite similar to the distributed case of bars considered for TOBA. In this case we have

$$
\delta \theta_{\mathrm{mech}}^{f r}(\omega) \simeq \frac{16}{a \omega_{f r}} \sqrt{\frac{\phi_{\mathrm{mech}}^{f r} k_{B} T^{f r}}{M_{f r} \omega}} .
$$

The parameters in (26) are related to the frame: $\phi_{\text {mech }}^{f r}$ is the mechanical loss angle of the frame material, $M_{f r}$ is the mass of the frame, $\omega_{f r}$ is the lowest mechanical angular resonant frequency: $\omega_{f r}=\pi u_{f r} / a$, and $u_{f r}$ is the speed of sound in the material of the frame. As we will see in Sec. III B below, the frame temperature $T^{f r}$ should be at the cryogenic operational range, although cooling of the barbells is not required.

\section{Brownian noise of the suspension}

As for the TOBA detectors, both terrestrial and orbital deployments are possible although the suspensions required are very different for these two cases. The angular fluctuations 


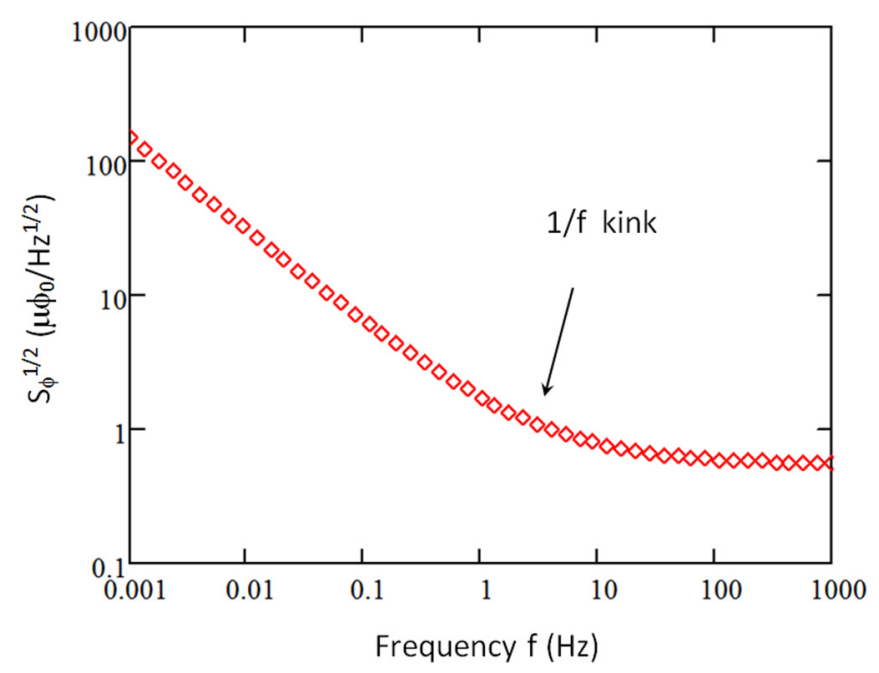

FIG. 4. Noise spectrum (28) of a highly sensitive SQUID current sensor based on sub-micrometer cross-type Josephson junctions taken at $T=4 \mathrm{~K}$ [55].

associated with the suspension can be expressed as [33]

$$
\delta \theta_{\text {sus }}(\omega)=\frac{\sqrt{4 \gamma k_{B} T}}{I \omega^{2}}
$$

where $\gamma$ is the damping factor. This factor depends on the suspension specifics. For the terrestrial mounting one can expect much larger values of $\gamma$ than for orbital placement, since the suspension should withstand much lower forces in the latter case.

\section{Signal detector (SQUID) noise}

The spectral noise of SQUIDs is conventionally determined by the function $S_{\phi}^{1 / 2}(f)$ [54]. The energy resolution $\delta E$ per bandwidth $\delta f$ of the SQUID is $\delta E / \delta f=S(f) /\left(2 L_{\text {input }}\right)$, where $L_{\text {input }}$ is the input inductance of the SQUID coil. To be detectable, the pulse energy $E_{\text {pulse }}$ should exceed the value $\delta E$, i.e., $E_{\text {pulse }} \geqslant\left[S(f) /\left(2 L_{\text {input }}\right)\right] \times \delta f$. In the so-called "quantum limit", $S(f) /\left(2 L_{\text {input }}\right) \simeq \hbar$, so that $E_{\text {pulse }} \geqslant \hbar \times \delta f$. If the GW-pulse delivers energy $E_{\text {pulse }}=E_{m}$ of which the half may be dynamically transferred to the circuit (impedance matching condition!), then, the condition $E_{m} \geqslant 2 \hbar \times \delta f$ should be fulfilled for detectability. Associating, as customarily done, the characteristic Fourier-frequency $\omega_{\mathrm{GW}}$ with the acquisition bandwidth $\delta f$, we arrive at Eq. (1). That means that the SQUID's "quantum limit", subject to Eq. (18), yields the ultimate energy resolution limit (ERL) discussed above. In reality, it is hard to reach the $\hbar$ limit. A more realistic example is 5 $\hbar$ SQUID [55] with input coil inductance $L_{\text {input }}=0.6 \mu H$ and a spectral noise function

$$
S_{\phi}^{1 / 2}(f)=0.55 \mu \phi_{0} / H z^{1 / 2} \times\left[1+\left(\frac{3 H z}{f}\right)^{0.7}\right],
$$

where $\phi_{0} \simeq 2 \times 10^{-15} \mathrm{~Wb}$ is the flux quantum. At $f=$ $100 \mathrm{~Hz}, S_{\phi}^{1 / 2}(f)=0.6 \mu \phi_{0} / H z^{1 / 2}$. Because of the $1 / f$ kink, the noise (28) is much greater at sub-Hz frequencies, as indicated in Fig. 4. However, as we will see below, this still offers a sufficiently good performance.

\section{Johnson-Nyquist noise in the circuit}

The spectrum of current noise in superconductors per unit bandwidth is $4 k_{B} T / R$, where $R$ is the resistance of the normal excitations in superconductors: $R=4 a \rho_{n} / S_{\text {wire }}$. Here, $\rho_{n}$ is the resistivity of the normal carriers in superconductors and $S_{\text {wire }}$ is the wire cross section. In finite-gap superconductors, $\rho_{n}$ is exponentially large because of exponential reduction of the number of unpaired electrons at $T \ll T_{c}$. Thus, the current noise is

$$
\left\langle J_{\text {noise }}\right\rangle=\sqrt{\frac{4 k_{B} T^{f r} S_{\text {wire }}}{4 \rho_{n} a} f} \times \exp \left(-\frac{0.88 T_{c}}{T^{f r}}\right),
$$

where we assumed a superconductor with the isotropic BCS energy gap $\Delta(T)=1.76 T_{c}$ and $T^{f r} \ll T_{c}$. Because of the gap isotropy, this noise exponentially dies out and becomes insignificant at low temperatures, as we will see from numerical analysis in Sec. III B [56].

\section{Shot noise of the magnetic field}

The shot noise will be picked up by the superconducting loops as a parasitic addition to the GW-generated signal. However, any variation of the magnetic field, which is not related to the rotation of the superconducting loops may be independently tracked by a third, immobile loop (which is not shown in Fig. 1) and digitally subtracted from the GW signal output.

Additionally, stray fields caused by thermal fluctuations of external objects, such as those due to the suspension of the barbells and the coils, may cause spurious signal pickups. Spurious signals can be caused also by charged cosmic ray particles crossing the superconducting loops. However, one should recall that in our design there are two active loops as in Fig. 1. When actuated by a GW, these loops generate equal but opposite sign signals so that a nonzero output is obtained by their subtraction. Meanwhile, stray fields will generate the same signals in the two loops, which will then cancel upon subtraction. Using this approach, we reached the theoretical Johnson-Nyquist noise level in laboratory modeling of the GW detector with the normal-metal loops [47].

\section{Newtonian noise}

Last but not least is the Newtonian noise. In our case, the presence of two barbells should assist in tracking their synchronous motions caused by external gravitating objects and, after appropriate calibration, digitally canceling out the related signals.

\section{B. Implementable device with useful strain sensitivity}

Let us find the parameters of a realistic system needed to achieve interesting strain sensitivity for an operational frequency in the $\mathrm{mHz}$ range, $f=0.001 \mathrm{~Hz}$. We will consider barbells with the sphere mass $M=4$ ton (in the case of $\mathrm{Pb}$, this corresponds to a radius $R=44 \mathrm{~cm}$ ). The barbell arms, made of fused silica, have length $r=50 \mathrm{~m}$. In this material the intrinsic mechanical loss angle is $\phi_{\text {mech }}^{b} \simeq 10^{-6}$ at $T=300 \mathrm{~K}$ [57]. For an orbital placement, this fused silica arm can be made of a tube with 2-mm wall thickness, and $3-\mathrm{cm}$ diameter, in which case its mass will be negligible 
$(<100 \mathrm{~kg})$ compared with the sphere mass $M$. Then from (25): $\delta \theta_{\text {mech }}^{b}(f=1 \mathrm{mHz})=2.8 \times 10^{-19} 1 / \mathrm{Hz}^{1 / 2}$ (in our design, the barbells operate at ambient conditions: we assume $T=$ $300 \mathrm{~K}$ ). For terrestrial arrangement, there should be a more sophisticated structure, like a bridge arch, fused from the same material, which may have significantly larger arm mass than that for the orbital deployment. In both cases, the mass of the barbell is expected to be much larger than that of the arms, and is comparable to the mass of the bar of the TOBA detector [33] ( 8 ton vs 7.6 ton). This suggests a torsion damping factor in our case comparable with that of TOBA, $\gamma^{\text {terr }} \sim 10^{-10} \mathrm{~N}$ m s. As was mentioned above, for an orbital instrument, this quantity could be significantly reduced. For our orbital instrument, we will conservatively assume $\gamma^{\text {orb }} \sim 10^{-11} \mathrm{~N} \mathrm{~m} \mathrm{~s}$. Then, for a noncooled $(T=300 \mathrm{~K})$ suspension we find from Eq. (27): $\delta \theta_{\text {sus }}^{\text {terr }}(f=1 \mathrm{mHz}) \simeq 5.2 \times 10^{-19} 1 / \mathrm{Hz}^{1 / 2}$ and $\delta \theta_{\text {sus }}^{\text {orb }}(f=1 \mathrm{mHz}) \simeq 1.6 \times 10^{-19} 1 / \mathrm{Hz}^{1 / 2}$. We will choose $B=10 \mathrm{~T}$ (corresponding to a superconducting magnet) and a niobium nitride $(\mathrm{NbN})$ alloy as a superconducting material of the loops in Fig. 1. This material will be deposited around the frame with $a=5 \mathrm{~cm}$ so as to be equivalent to, and yield the same inductance as, a superconducting wire of diameter $d \simeq 1 \mu \mathrm{m}$. Since this frame should be cryogenically cooled, sapphire is an ideal material for it. We will assume the frame with a mass $M_{f r} \sim 3 \mathrm{~kg}$ is at $T=0.3 \mathrm{~K}$. Also, all of the superconducting electronics will reside in the same cryostat. Interestingly, the loss angle of monolitic sapphire at low frequencies and temperatures may be much lower than that of fused silica at $300 \mathrm{~K}$. From the KAGRA study [58], we find that for sapphire $\phi_{\text {mech }}^{f r} \simeq 10^{-8}$ at $T=0.3 \mathrm{~K}$ and $f=1 \mathrm{mHz}$. Also, $u^{\text {sapphire }} \approx 10^{4} \mathrm{~m} / \mathrm{s}$. Then, from Eq. (26): $\delta \theta_{\text {mech }}^{f r}(f=$ $1 \mathrm{mHz})=2.4 \times 10^{-19} 1 / \mathrm{Hz}^{1 / 2}$. The total mechanical noise from these three contributions is

$$
\begin{aligned}
\delta \theta_{\text {mech }}^{\text {tot }} & =\sqrt{\left(\delta \theta_{\text {mech }}^{b}\right)^{2}+\left(\delta \theta_{\text {mech }}^{f r}\right)^{2}+\left(\delta \theta_{\text {sus }}\right)^{2}} \\
& \simeq(5-7) \times 10^{-19} / \mathrm{Hz}^{1 / 2} \text { at } f=1 \mathrm{mHz},
\end{aligned}
$$

where the smaller and larger values correspond to the orbital and terrestrial placements. In accordance to Eqs. (15) and (16), this yields a flux noise

$$
\begin{aligned}
\delta \hat{\Phi}_{\text {mech }}^{\text {tot }} \equiv \delta \Phi_{\text {mech }}^{\text {tot }} / \sqrt{f} & =B a^{2} \delta \theta_{\text {mech }}^{\text {tot }} \\
& \simeq(7-9) \mu \phi_{0} / \mathrm{Hz}^{1 / 2} .
\end{aligned}
$$

This flux noise caused by thermodynamic fluctuations in the mechanical system should be complemented by the noise in electrical circuits, including the electric current detector noise. To minimize the noise, the impedance matching of the frame loop with that of the SQUID coil is required. For the SQUID system quoted above (see Fig. 4), the inductance of the input coil will be matched $\left(L_{\text {loop }} \approx L_{\text {coil }} \approx 0.5 \mu \mathrm{H}\right)$ at the chosen rectangular loop size $(a \simeq 5 \mathrm{~cm})$ and the wire diameter $(d=$ $1 \mu \mathrm{m})$. At this choice of parameters, no flux transformer is required and the SQUID coil can be directly integrated with our loop. To simplify the situation, one can consider superconducting loop as (a gigantic) SQUID itself. The corresponding flux noise of the SQUID system at $T=4 \mathrm{~K}$, in accordance to (28), is then (see Fig. 4):

$$
S_{\phi}^{1 / 2}(f=1 \mathrm{mHz})=150 \mu \phi_{0} / \mathrm{Hz}^{1 / 2} .
$$

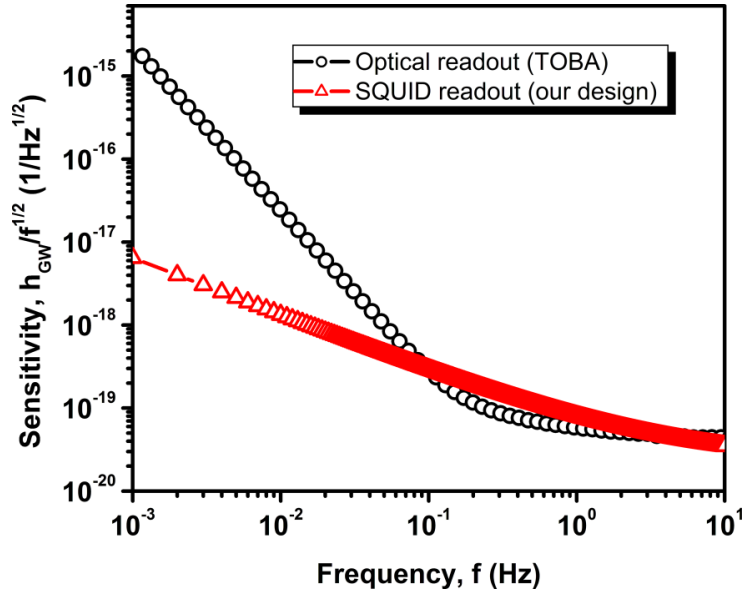

FIG. 5. Comparison of strain sensitivities of GW sensors based on optical interferometric and superconducting quantum interferometric readouts.

This SQUID noise is generated by the normal resistance $R_{J J}$ of its Josephson junctions. This resistance has the spectral function $S_{\phi} \approx 4 k_{B} T / R_{J J}$. Typically, $R_{J J}$ is temperature independent, and at temperature reduction from $T=4 \mathrm{~K}, S_{\phi}$ scales linearly with $T$ [54,59], i.e., the spectral function $S_{\phi}$ reduces linearly with $T$. At $T=0.3 \mathrm{~K}$ operational temperature, one should expect $S_{\phi}^{1 / 2}(f=1 \mathrm{mHz}) \approx 40 \mu \phi_{0} / \mathrm{Hz}^{1 / 2}$. This means that the SQUID noise, though smaller than in Eq. (32), is the dominating factor in this particular design:

$$
\begin{aligned}
\delta \hat{\Phi}^{\text {tot }} & \simeq \delta \hat{\Phi}^{\text {SQUID }}+\delta \hat{\Phi}_{\text {mech }}^{\text {tot }} \\
& \leqslant 50 \mu \phi_{0} / \mathrm{Hz}^{1 / 2} \text { at } f=1 \mathrm{mHz} .
\end{aligned}
$$

We did not include in (33) a flux noise caused by the Johnson-Nyquist fluctuations (29): $\left\langle\Phi_{J-N}\right\rangle=L_{\text {coil }}\left\langle J_{\text {noise }}\right\rangle$ because it is negligibly small. Indeed, choosing $\mathrm{NbN}$ as a superconducting material with $T_{c}=15 \mathrm{~K}$ and normal state resistivity for the deposited wire $\rho_{n} \simeq 10^{-7} \mathrm{Ohm}-\mathrm{m}$, we find at $T^{f r}=0.3 \mathrm{~K}$ :

$$
\delta \hat{\Phi}_{J-N}(f=1 \mathrm{mHz}) \simeq 10^{-18} \mu \phi_{0} / \mathrm{Hz}^{1 / 2},
$$

which is negligibly small compared with (33) and can be safely neglected.

To conclude this discussion, we should notice that the mechanical resonant frequency of the system below which the performance degrades, is

$$
f_{\text {res }} \approx 0.6 \mathrm{mHz}
$$

as follows from Eq. (19). The corresponding amplitude of $h_{\mathrm{GW}}$ with a bandwidth equal to the detected frequency is shown in Fig. 5.

\section{Advantages of our approach}

GEFEST and TOBA have many similar mechanical properties, including suspension, certain noise mitigation, resonant frequency limitations, etc. They will be compactly arranged in space. As such, GEFEST will be advantageous for the detection of $\mathrm{GW}$ for lower frequencies. For comparable mechanical parameters it is interesting to compare the sensitivity of superconducting vs optical interferometric detection. As 


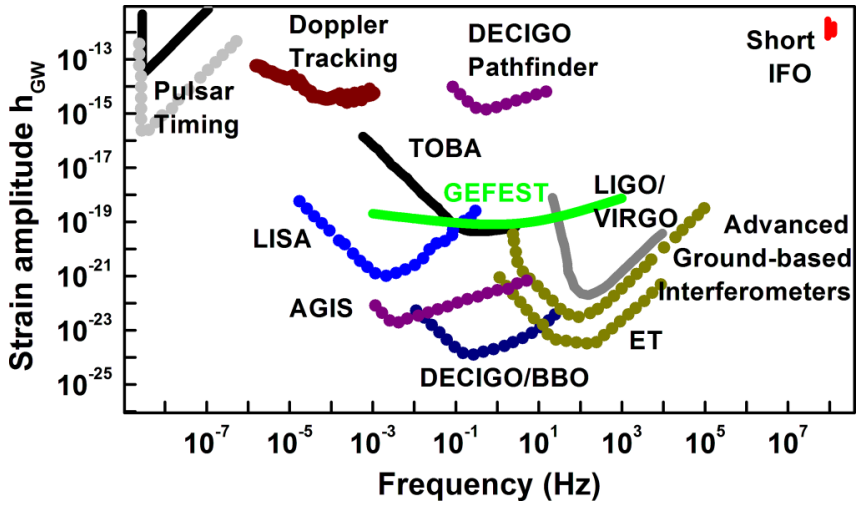

FIG. 6. Comparison of sensitivity of the GEFEST detector with the current (bold curves) and projected (dotted curves) GW detectors [33]. The GEFEST parameters correspond to those mentioned in the section devoted to the implementable device with interesting strain sensitivity. The characteristic amplitudes of GWs are defined as the amplitude with a bandwidth equal to the observation frequency.

follows from Figs. 5 and 6, the superconducting approach prevails at the low- $\mathrm{mHz}$ range of GW frequencies.

\section{DISCUSSION}

The above described advantage of the superconducting design over the optical interferometric method is due to the fact that we have no laser or mirrors; the noise associated with the latter causes the upturn of the TOBA curve in Figs. 5 and 6 . The higher sensitivity of the GEFEST design requires cryogenic cooling. As we have shown, this is in principle achievable. The results shown in Fig. 6 for GEFEST are obtained without any signal upconversion or heterodyning. The $1 / f$ noise is explicitly taken into account in the SQUID noise figure (Fig. 4). Sometimes, upconversion/heterodyning has additional advantages (see, e.g., [31-33]). In our case, if necessary, the upconversion/heterodyning can be achieved electronically without mechanical rotation. Importantly, cryogenic cooling is only necessary for the superconducting transducer of the detector. Only the relatively small loops $\left(5 \times 5 \mathrm{~cm}^{2}\right.$ in the case of the chosen parameters) need to be cooled down to $300 \mathrm{mK}$; the superconducting magnet can operate at $4 \mathrm{~K}$ or even higher.

Higher sensitivity of the GEFEST can be achieved either with larger loops, or with multiturn small loops. Both will require larger barbell masses to generate more mechanical energy for harvesting. Compact cryostats assume multiturn rather than large coils. This issue is discussed in some detail in the Appendix.

The cryostats for the barbell-related loops/coils, as well as for the superconducting magnet are spatially separated from each other, so that the magnet with its own cryostat is immobile, while the cryostats surrounding each superconducting loop (with the relevant superconducting electronics enclosed) are moving jointly with the loops (and the barbells), so that a lossless transfer of mechanical motion inside the cryostat is not required.

As mentioned in [33], the potential sensitivity of laser interferometric LIGO-type and TOBA-type detectors is ultimately determined by $h_{\mathrm{GW}}^{\mathrm{ERL}} \propto 1 / \sqrt{M r^{2}}$, with an arm length $r$ and a mirror mass $M$. This ultimate sensitivity, for LIGO-type interferometric instruments, is hard to achieve by increasing $M$, since other noise-related parameters like mirror surface imperfections/fluctuations become relevant [15]. While cooling can help to reduce such noise, it looks impossible to have cryogenically cooled mirrors or bars with a very large mass (say, $M=100$ ton). Thus the extension of $r$ is the major direction of future development for LIGO-type interferometric instruments. In our case, very long arms are not plausible, and barbells themselves do not need cryogenic cooling. Thus, even the mass $M=100$ ton does not look like a limit, and its increase can enable increasing the sensitivity of future superconducting instruments. Correspondingly, assuming appropriately chosen parameters, various approaches may have competitive performance utilizing different values of mechanical parameters: either $M$ or $r$. The sensitivity shown in Fig. 6, for barbells with 10 ton mass and $50 \mathrm{~m}$ arms, may be significantly improved by varying these parameters. To conclude this section, we remind that, as described in Fig. 3, the real instruments should have a "triangular" design for the barbell arms. In accordance with Eq. (25), this will increase the thermomechanical noise by at least a factor of $\sqrt{2}$. However, as was shown in Sec. III B, the dominant noise contribution comes from the SQUID circuitry, so the replacement of a simple rod by a triangle will not degrade the GEFEST performance anyhow significantly.

\section{SUMMARY}

Above we discussed some ideas for utilizing the motion of Cooper pairs in superconductor-based GW antennas, which can complement and be competitive with interferometric devices. A unique feature of this technique is the efficient transfer of mechanical energy received from a GW into magnetic flux via superconducting circuits, and subsequent detection of this flux by superconducting electronics. The role of the mechanical parameters of the detector in harvesting the energy of GWs, as well as that of various thermodynamically fundamental noise sources has been examined. Based on general considerations, a specific practically interesting case is considered. It can be implemented using currently available technologies both terrestrially and orbitally. Assuming future improvements in technologies, it will become possible to achieve phenomenal sensitivities with locally arranged orbital instruments. These instruments may become viable alternatives to interferometric devices.

\section{ACKNOWLEDGMENTS}

We would like to express our gratitude to Y. Aharonov, P. Marronetti, A. Kadin, D. Kirichenko, V. Gurzadyan, I. Ciufolini, A. Paolozzi, H. J. Paik, J. Luine, A. Arvanitaki, R. Adhikari, N. Christensen, J. Isenberg, J. Smith, D. Tanner, R. Adler, P. Pearle, A. Kuklov, P. Taylor, U. Leonhardt, D. Van Vechten, and D. Struppa for their support and useful comments and discussions. We also would like to acknowledge very constructive discussions with anonymous reviewers of Phys. Rev. Research which greatly improved our consideration. This research is supported in part by the ONR Grants No. N00014-20-1-2442 and No. N00014-19-1-2265. 

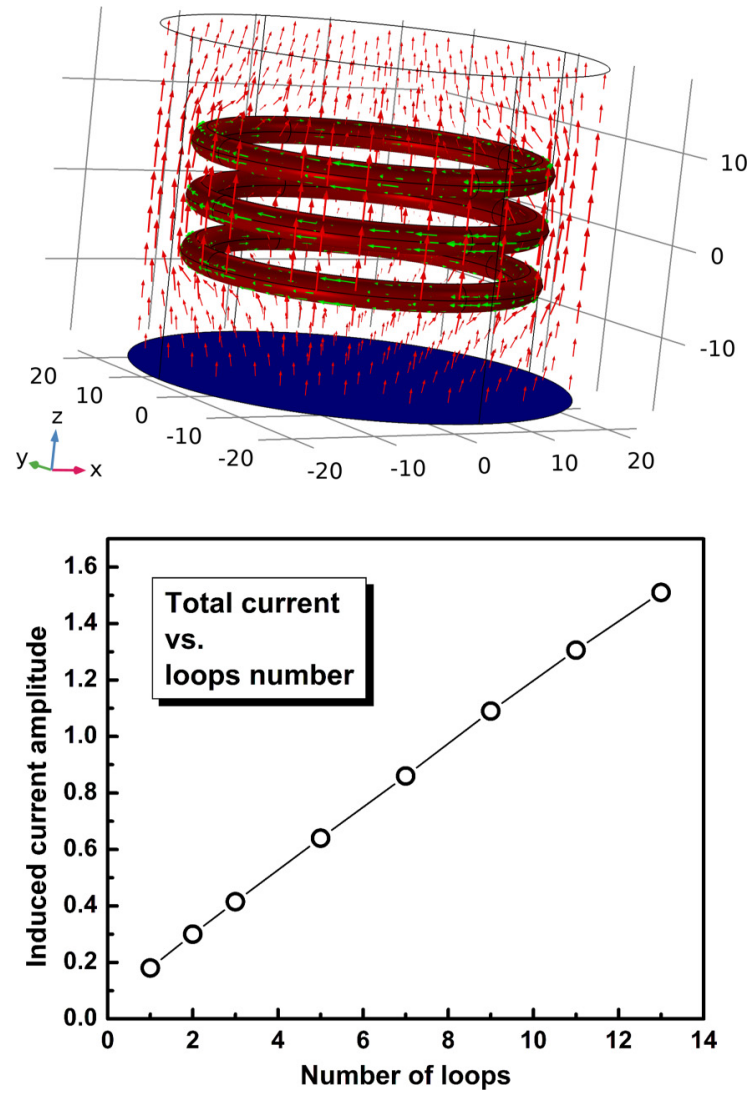

FIG. 7. In case of superconducting loops arranged at a distance (top panel) the induced current in a good approximation is a linear function of their number (bottom panel). The distance between the loops is taken equal to the wire diameter. Red arrows indicate magnetic-flux density lines (induction vector B). Green arrows indicate current density $\mathbf{j}$. We confirmed linearity for up to 13 parallel rings. Practical computational limits prevent going to much higher number of rings.

\section{APPENDIX: TRANSDUCER LOOP STACKING}

Multiloop design can facilitate smaller loop and frame sizes and higher sensitivity. However, it is not very obvious how to organize the loops so as to optimize the performance. Here we address this topic.

\section{Connecting loops in series: superconducting coils}

Variation of the flux as a function of time $\Phi(t)=$ $\delta \phi \sin (\omega t)$ generates electromotive force in the loop:
$\mathcal{E}=-d \Phi / d t$, resulting in a voltage difference $V=\mathcal{E}$ between the ends of an open loop. With $N$ loops constituting a coil, the voltage becomes $V_{N}=N V$. For a single superconducting loop with inductance $L$, the current equals $J=$ $V /(L \omega)$, while for a coil, the current is $N$ times smaller: $J_{N}=V_{N} /\left(L_{N} \omega\right)=J / N$ since the coil's inductance is $L_{N}=$ $N^{2} L$. Thus the maximum flux transferrable to the detector is $J_{N} L_{N}=N L J=N \delta \phi$. However, the transferred energy is the same as with the single-loop transducer: $(N \delta \phi)^{2} /\left(2 L_{N}\right)=$ $\delta \phi^{2} / 2 L$. Thus one cannot improve signal/noise ratio using a coil since the transferred energy is not enhanced relative to the energy in the SQUID's noise.

\section{The case of parallel loops}

At first glance, parallel action of loops does not deliver benefits either. Indeed, in the case of $N$ independent loops we need to take into account the self inductances $L_{i}(\equiv L)$ of each loop and the mutual inductances $M_{i j}(i, j=1,2, \ldots, N ; i \neq$ $j)$. Suggesting that the $N$ loops screen the flux $\delta \phi$ jointly, one can deduce that the current in each loop will be $J_{i}=$ $\delta \phi /(N L)=J_{N}$. Then the total transferred energy will be

$$
\begin{aligned}
\sum_{i} \frac{L_{i} J_{i}^{2}}{2}+\sum_{i, j} M_{i j} J_{i}^{2} & =\frac{1}{2} N L J_{N}^{2}+\frac{1}{2} N(N-1) M J_{N}^{2} \\
& =\frac{1}{2} N^{2} L J_{N}^{2}=\frac{1}{2} L J^{2}=\frac{\delta \phi^{2}}{2 L},
\end{aligned}
$$

where a perfect coupling between the loops is assumed, so that $M_{i j}=M=L$. Thus the the total transduced energy is the same as with the $N$-turn coil, i.e., is equal to single loop energy. Fortunately, it is possible to find a loophole in the above argument. Two assumptions made therein can be questioned: equating the mutual and self inductances, and the joint screening of the flux when there is a distance between the loops (which makes them physically different from an ideal coil). Precise results for $N$ separated parallel loops are obtained from a finite element COMSOL modeling using the code described in the Supplemental Material [61]. The results are shown in Fig. 7.

In the first approximation, the total current (Fig. 7) is the sum of individual currents, and the presence of loops in this arrangement has almost no effect on the individual loop current. This means that each loop can be associated with its individual SQUID detector (as was mentioned in the main text, the current loops themselves may be the SQUIDs). Then the SQUIDs outputs can be digitally summed up so as to enhance the signal/noise ratio by a factor of $N^{1 / 2}$.
[1] M. E. Gertsenshtein and V. I. Pustovoit, On the detection of low frequency gravitational waves, Sov. Phys. JETP-USSR 16, 433 (1963) [ Zh. Eksp. Teor. Fiz. 43, 605 (1963)].

[2] B. P. Abbott et al. (LIGO Scientific Collaboration and Virgo Collaboration), Observation of Gravitational Waves from a Binary Black Hole Merger, Phys. Rev. Lett. 116, 061102 (2016).

[3] B. P. Abbott et al. (LIGO Scientific Collaboration and Virgo Collaboration), GW151226: Observation of Gravitational
Waves from a 22-Solar-Mass Binary Black Hole Coalescence, Phys. Rev. Lett. 116, 241103 (2016).

[4] T. Akutsu et al. (KAGRA Collaboration), KAGRA: 2.5 generation interferometric gravitational wave detector, Nat. Astron. 3, 35 (2019).

[5] M. Punturo et al., The Einstein telescope: A third-generation gravitational wave observatory, Classical Quant. Grav. 27, 194002 (2010). 
[6] B. P. Abbott et al., Exploring the sensitivity of next generation gravitational wave detectors, Classical Quant. Grav. 34, 044001 (2017).

[7] J. Miller, L. Barsotti, S. Vitale, P. Fritschel, M. Evans, and D. Sigg, Prospects for doubling the range of advanced LIGO, Phys. Rev. D 91, 062005 (2015).

[8] The Virgo Collaboration. Advanced Virgo Plus Phase IDesign Report; Technical Report VIR-0596A-19; 2019, https: //tds.virgo-gw.eu/q1/?c=14430.

[9] N. Seto, S. Kawamura, and T. Nakamura, Possibility of Direct Measurement of the Acceleration of the Universe Using $0.1 \mathrm{~Hz}$ Band Laser Interferometer Gravitational Wave Antenna in Space, Phys. Rev. Lett. 87, 221103 (2001).

[10] S. Kawamura et al., The Japanese space gravitational wave antenna - DECIGO, J. Phys.: Conf. Ser. 122, 012006 (2008).

[11] P. Amaro-Seoane et al., Laser interferometer space antenna, arXiv:1702.00786.

[12] J. Weber, General Relativity and Gravitational Waves, Interscience Manuals Series (Interscience Publishers, New York, 1961).

[13] J. Weber, Gravitational-Wave-Detector Events, Phys. Rev. Lett. 20, 1307 (1968).

[14] P. E. Michelson, Gravitational radiation detectors, in 100 Years of Superconductivity, edited by H. Rogalla and P. H. Kes (Taylor \& Francis, London, 2011), p. 369.

[15] P. R. Saulson, Fundamentals of Interferometric Gravitational Wave Detectors (World Scientific, Singapore, 1994).

[16] R. P. Giffard, Ultimate sensitivity limit of a resonant gravitational wave antenna using a linear motion detector, Phys. Rev. D 14, 2478 (1976).

[17] "Linear" term should be understood here as proportionality of the detector output vs the input signal.

[18] M. W. Mitchell and S. Palacios Alvarez, Colloquium: Quantum limits to the energy resolution of magnetic field sensors, Rev. Mod. Phys. 92, 021001 (2020).

[19] Indeed, in accordance to the quantum uncertainty principle, during the measurement time $\tau$ the energy can be measured with an accuracy $\delta E \geqslant \hbar / \tau$. To be measurable, the energy pulse delivered to the detector should exceed this value: $E \geqslant$ $\delta E \geqslant \hbar / \tau$. At dynamic energy transfer, because of impedance matching, only $50 \%$ of energy is possible to transfer to the detector. For transient pulses it is customary to consider that the measurement time should not be shorter than the inverse main Fourier harmonics range of the signal: $\tau \sim 1 / \omega_{\mathrm{GW}}$ which justifies approximate validity of Eq. (1) for wideband detectors.

[20] W. H. Press, and K. S. Thorne, Gravitational-wave astronomy, Annu. Rev. Astron. Astrophys. 10, 335 (1972).

[21] C. W. Misner, K. S. Thorne, and J. A. Wheeler, Gravitation (W.H. Freeman, San Francisco, 1973).

[22] B. F. Schutz, A First Course in General Relativity (Cambridge University Press, Cambridge, 1985).

[23] In case of a general polarization of the $\mathrm{GW}$, the response is a simple superposition.

[24] S. W. Hawking, Perturbations of an expanding universe, Astrophys. J. 145, 544 (1966).

[25] P. Abramian-Barco and A. Gulian, The numerical solutions of Hawking's equations for gravitational waves in the evolutionary universe, in Gravitational Waves: Explorations, Insights and Detection, edited by I. Carson (Nova Science Publishers, New York, 2017) pp. 1-35.
[26] R. L. Forward, Multidirectional, multipolarization antennas for scalar and tensor gravitational radiation, Gen. Relativ. Gravit. 2, 149 (1971)

[27] R. L. Forward, Review of artificial satellite gravity gradiometer techniques for geodesy, in The Use of Artificial Satellites for Geodesy and Geodynamics (Defense Technical Information Centers, 1974), p. 157.

[28] H. A. Chan, M. V. Moody, and H. J. Paik, Superconducting gravity gradiometer for sensitive gravity measurements. II. Experiment, Phys. Rev. D 35, 3572 (1987).

[29] M. V. Moody, H. J. Paik, and E. R. Canavan, Three-axis superconducting gravity gradiometer for sensitive gravity experiments, Rev. Sci. Instrum. 73, 3957 (2002).

[30] V. B. Braginsky, Y. B. Zel'dovich, and V. N. Rudenko, Reception of gravitational radiation of extraterrestrial origin, Sov. Phys. JETP Lett. 10, 280 (1969) [Zh. Eksp. i Teor. Fiz. Pis'ma, 10, 437 (1969)].

[31] V. B. Braginsky and V. S. Nazarenko, On the detection of gravitational radiation from some astronomical sources with the heterodyne detector, in Proc. Conf. on Experimental Tests of Gravitational Theories, edited by R. Davis (California Institute of Technology, Pasadena, 1971) p. 45.

[32] Unpublished work (1969), cited by Ref. [31], see also C.W. Misner, K. S. Thorne, and J. A. Wheeler, Gravitation (W. H. Freeman and Company, San Francisco, 1973), p. 1017.

[33] M. Ando, K. Ishidoshiro, K. Yamamoto, K. Yagi, W. Kokuyama, K. Tsubono, and A. Takamori, Torsion-Bar Antenna for Low-Frequency Gravitational-Wave Observations, Phys. Rev. Lett. 105, 161101 (2010).

[34] A. Gulian, J. Foreman, V. Nikoghosyan, L. Sica, J. Tollaksen, and S. Nussinov, Superconducting antenna concept for gravitational wave radiation, arXiv:1111.2655.

[35] C. G. Darwin, The inertia of electrons in metals, Proc. R. Soc. London A 154, 61 (1936).

[36] S. M. Kogan, Does an electron fall in a metallic pipe? Phys. Usp. 14, 658 (1972) [Usp. Fiz. Nauk, 105, 157 (1971)].

[37] I. M. Tsidil'kovskii, Electrons and holes in an inertial-force field, Sov. Phys. Usp. 18, 161 (1975) [Usp. Fiz. Nauk, 115, 322 (1975)].

[38] B. Cabrera and M. E. Peskin, Cooper-pair mass, Phys. Rev. B 39, 6425 (1989).

[39] A. Gulian, J. Foreman, V. Nikoghosyan, L. Sica, J. Tollaksen, and S. Nussinov, Superconducting antenna concept for gravitational wave radiation, arXiv:1111.2655.

[40] R. C. Tolman and T. D. Stewart, The electromotive force produced by the acceleration of metals, Phys. Rev. 8, 753 (1916).

[41] A. J. Dessler, F. C. Michel, H. E. Rorschach, and G. T. Trammell, Gravitationally induced electric fields in conductors, Phys. Rev. 168, 737 (1968).

[42] L. I. Schiff, Gravitation-Induced Electric Field near a Metal. II, Phys. Rev. B 1, 4649 (1970).

[43] A. Gulian, J. Foreman, V. Nikoghosyan, L. Sica, J. Tollaksen, and S. Nussinov, Superconducting antenna concept for gravitational wave radiation, arXiv: $1111.2655 \mathrm{v} 3$.

[44] A. Gulian, J. Foreman, V. Nikoghosyan, L. Sica, J. Tollaksen, and S. Nussinov, Superconducting antenna concept for gravitational wave radiation, arXiv:1111.2655v4.

[45] F. London, Superfluids, Vol. 1: Macroscopic Theory of Superconductivity (Dover Publications, New York, 1961). 
[46] A. Gulian, Shortcut to Superconductivity: Superconducting Electronics via COMSOL Modeling (Springer International Publishing, Cham, 2020).

[47] A. Gulian, J. Foreman, V. Nikoghosyan, L. Sica, C. Burdette, J. Tollaksen, and S. Nussinov, Rotational superconducting gravitational wave detectors based on Cooper-pair electronic transducers, IEEE Trans. Appl. Supercond. 29, 1 (2019).

[48] By that we mean that the flux in the other loop will have similar amplitude but opposite sign, the current of similar amplitude will flow in opposite direction, etc.

[49] Hereafter we drop the term $\gamma(\delta \dot{\theta})$ related to the suspension in the equations of motion. It is quite similar to the case of TOBA [33]. However, we will take into account the noise related with this $\gamma$-term when discussing the suspension-related noise. At orbital deployment, GEFEST can be designed to operate in absence of any suspension.

[50] F. W. Grover, Inductance Calculations : Working Formulas and Tables (D. Van Nostrand, New York, 1946).

[51] In Eq. (60) on page 60 in [50], the coefficient 0.008 should be replaced by $2 \mu_{0} / \pi$ in the SI units.

[52] H. B. Callen and T. A. Welton, Irreversibility and generalized noise, Phys. Rev. 83, 34 (1951).

[53] P. R. Saulson, Thermal noise in mechanical experiments, Phys. Rev. D 42, 2437 (1990).
[54] J. Clarke, Squid fundamentals, in SQUID Sensors: Fundamentals, Fabrication and Applications, edited by H. Weinstock (Springer Netherlands, Dordrecht, 1996), p. 13.

[55] M. Schmelz, V. Zakosarenko, T. Schönau, S. Anders, J. Kunert, M. Meyer, H.-G. Meyer, and R. Stolz, A new family of field-stable and highly sensitive SQUID current sensors based on sub-micrometer cross-type Josephson junctions, Supercond. Sci. Technol. 30, 074010 (2017).

[56] For the best possible normal-metal loops one will have a voltage Johnson-Nyquist noise which, as simple estimates are providing, is much stronger than the GW-induced signal even in deeply-cooled case.

[57] Mechanical Loss in Silica and Silica/Alumina Coatings, https: //dcc.ligo.org/public/0037/G080302/000/G080302-00.pdf.

[58] T. Uchiyama, T. Tomaru, D. Tatsumi, S. Miyoki, M. Ohashi, K. Kuroda, T. Suzuki, A. Yamamoto, and T. Shintomi, Mechanical quality factor of a sapphire fiber at cryogenic temperatures, Phys. Lett. A 273, 310 (2000).

[59] Actually, the linearity was demonstrated down to $150 \mathrm{mK}$ [60].

[60] F. C. Wellstood, C. Urbina, and J. Clarke, Low-frequency noise in dc superconducting quantum interference devices below $1 \mathrm{~K}$, Appl. Phys. Lett. 50, 772 (1987).

[61] See Supplemental Material at http://link.aps.org/supplemental/ 10.1103/PhysRevResearch.3.043098 for technical details of the model. It also contains the evidence that the current amplitude does not depend on the speed of the external flux variation. 